Revue internationale P.M.E.

Économie et gestion de la petite et moyenne entreprise

\title{
Mesure de la sophistication des technologies de l'information dans les PME
}

\section{Guy Paré et Louis Raymond}

Volume 4, numéro 1, 1991

URI : https://id.erudit.org/iderudit/1008026ar

DOI : https://doi.org/10.7202/1008026ar

Aller au sommaire du numéro

Éditeur(s)

Presses de l’Université du Québec

ISSN

0776-5436 (imprimé)

1918-9699 (numérique)

Découvrir la revue

Citer cet article

Paré, G. \& Raymond, L. (1991). Mesure de la sophistication des technologies de l'information dans les PME. Revue internationale P.M.E., 4(1), 81-106.

https://doi.org/10.7202/1008026ar

\section{Résumé de l'article}

Le présent article présente une étude méthodologique relative au concept de sophistication de la technologie de l'information dans le contexte spécifique de la petite et moyenne entreprise. Si l'on désire identifier les effets de la technologie de l'information sur la structure, la stratégie ou encore la performance de ces organisations, if faut être capable de la caractériser dans un but d'opératio- nalisation en tant que variable indépendante, dépendante ou modératrice à l'intérieur d'un cadre conceptuel de recherche. Une première étape consiste donc à élaborer et à valider un instrument de mesure ayant trait au niveau de sophistication de la technologie de l'information dans les PME. Pour cet objectif, une étude empirique fut réalisée auprès de 101 PME manufacturières québécoises. 


\title{
Mesure de la sophistication des technologies de l'information dans les PME
}

\author{
Guy PARÉ* \\ École des Hautes Études Commerciales de Montréal \\ Louis RAYMOND** \\ Université du Québec à Trois-Rivières
}

\begin{abstract}
RÉSUMÉ
Le présent article présente une étude méthodologique relative au concept de sophistication de la technologie de l'information dans le contexte spécifique de la petite et moyenne entreprise. Si l'on désire identifier les effets de la technologie de l'information sur la structure, la stratégie ou encore la performance de ces organisations, il faut être capable de la caractériser dans un but d'opérationalisation en tant que variable indépendante, dépendante ou modératrice à l'intérieur d'un cadre conceptuel de recherche. Une première étape consiste donc à élaborer et à valider un instrument de mesure ayant trait au niveau de sophistication de la technologie de l'information dans les PME. Pour cet objectif, une étude empirique fut réalisée auprès de 101 PME manufacturières québécoises.
\end{abstract}

\section{ABSTRACT}

This article presents a methodological study regarding the concept of information technology sophistication in the specific context of small and medium-sized businesses (SMBs). If one wishes to identify the effects of IT on organizational

* Guy Paré est détenteur d'un M.Sc. de l'École des Hautes Études Commerciales de Montréal. Il est chargé d'enseignement au Service de l'enseignement des méthodes quantitatives et des systèmes d'information à l'École des Hautes Études Commerciales de Montréal. Il est membre du Groupe de recherche en Systèmes d'information (GRESI) de l'École des Hautes Études Commerciales. Adresse : École des Hautes Études Commerciales, Service des MQSI, 5255, avenue Decelles, Montréal (QC), H3T 1 V6.

** Louis Raymond est professeur titulaire en Systèmes d'information à l'Université du Québec à Trois-Rivières. Il est détenteur d'un Ph.D. en administration de l'École des hautes études commerciales de Montréal. Ses articles ont paru dans divers périodiques tels que MIS Quarterly, Journal of Management Information Systems, Database, American Journal of Small Business et Journal of Small Business Managenent. Le professeur Raymond est également auteur de livres en systèmes d'information et en bureautique. 
structure, strategy and performance, one must be able to characterize IT for operationalization purposes as a construct within a research framework. An initial step consists in developing and validating an instrument designed to measure an organization's level of sophistication in the use and management of IT. Such an instrument was built for the specific context of small and mediumsized enterprises and tested with a sample of 101 manufacturing organizations.

\section{RESUMEN}

Este articula presenta un estudio metodológico sobre el concepto de la sofisticación de la tecnología de la información en el contexto particular de la pequeña y mediana empresa. Si deseamos identificar los efectos de la tecnología de la información sobre la estructura, la estrategia y la performance de estas organizaciones, es necesario que la podamos caracterizar, con el objectivo de operacionalizarla al interior de un marco conceptual de investigación. La primera etapa consiste entonces a elaborar y a validar un instrumento de medida del grado de sopfisticación de la tecnología de la información en estas organizaciones. Tal instrumento que desarrollado a partir de une estudio empírico realizado en 101 empresas manufactureras de Quebec.

\section{Introduction}

Aux prises avec un niveau croissant de complexité et d'incertitude dans leur environnement, les petites et moyennes entreprises investissent actuellement de plus en plus dans la technologie de l'information pour tenter d'accroître leur productivité et d'améliorer l'efficacité du processus décisionnel de leurs dirigeants. L'informatique d'abord, puis la télématique, la bureautique et la productique sont des technologies de l'information (TI) qui tendent à être liées de plus en plus à la structure (Raymond, 1990), à la stratégie (Miller, 1987) et à la performance des PME (Bergeron et Buteau, 1988).

Un des problèmes fondamentaux des chercheurs en systèmes d'information et en théorie des organisations est de nature méthodologique. En fait, si l'on veut identifier les effets de la technologie de l'information sur l'organisation, il faut être capable de définir cette TI en tant que construit et caractériser cette dernière dans un but d'opérationalisation en tant que variable indépendante, dépendante ou modératrice dans un cadre conceptuel de recherche. Or, il n'existe à ce jour aucun instrument reconnu de mesure de la sophistication de la TI qui soit fidèle et valide pour fins d'études empiriques et de diagnostic, et ce, à la fois pour les entreprises de grande et de petite dimension (Benbasat et al., 1984). Un instrument de mesure de la technologie de l'information dans une PME devra donc en caractériser la nature et positionner l'organisation sur chacune des dimensions de cette technologie, c'est-à-dire établir l'existence de profils types de sophistication (Raymond, 1988). 
Les PME constituent un terrain initial propice d'étude en raison de leur spécificité sur le plan structurel, stratégique et technologique (Mintzberg, 1979; Julien et Marchesnay, 1988), ce qui permet de déterminer clairement un ensemble de caractéristiques descriptives de l'utilisation et de la gestion de la TI dans ce contexte. La documentation normative relative à l'utilisation et la gestion de la TI dans les petites et moyennes entreprises s'avère particulièrement abondante. Toutefois, les recommandations qui y sont faites découlent, trop souvent, de réflexions personnelles et ne reposent que très rarement sur des études empiriques. Ce constat motive la nécessité d'élaborer et de valider un instrument de mesure du degré de sophistication de la technologie de l'information dans les PME. Ultérieurement, l'utilisation de cet instrument pour fins de diagnostic organisationnel permettra de mieux situer une entreprise par rapport aux autres, par exemple celles appartenant à un même secteur d'activités, sur le plan de son utilisation et de sa gestion de la TI, et ainsi de définir les solutions proposées en fonction d'une meilleure connaissance de l'entreprise.

\section{De l'évolution des SIO à la sophistication de la TI}

Le modèle de Nolan (1973) fut le premier à tenter de conceptualiser l'évolution de l'informatique dans les organisations. Dans un contexte d'adoption de la TI dans les entreprises, le concept d'évolution consiste à déterminer ainsi qu'à planifier les différentes phases de croissance de l'informatique. Un des objectifs poursuivis par Nolan consistait alors à expliquer la relation analytique entre un stade et le stade précédent ou le stade suivant. Examinée sous cet angle, la notion d'évolution des SIO représente un concept dynamique mettant au premier plan une analyse de type causale voulant que seul l'apprentissage organisationnel permette le passage d'un stade d'évolution à l'autre et que les différents stades ne puissent être sautés, puisque l'expérience de la précédente est nécessaire au passage à la suivante.

Quoique contesté (King et Kraemer, 1984 ; Benbasat et al., 1984), le modèle de Nolan constitue encore l'une des principales références de base pour les chercheurs qui étudient la dynamique des systèmes d'information. La première version du modèle, qui se veut descriptive, présente quatre stades de l'évolution de l'informatique dans les organisations, soit l'initiation, la prolifération, le contrôle et l'intégration.

La phase d'initiation de l'informatique dans l'organisation permet de satisfaire les besoins informationnels de base de celle-ci, l'utilisation de l'ordinateur étant ainsi limitée à quelques applications particulières. Peu de solutions sont apportées à la fois aux problèmes causés par le rôle de l'ordinateur en tant qu'agent de changement et à ceux associés à la sous-utilisation de la capacité de 
traitement de l'ordinateur. Lors de la seconde phase, les demandes de développement d'applications et d'acquisition de nouveau matériel affluent, entraînant du même coup une forte hausse des dépenses en matériel, en logiciel et en personnel. Cette période de croissance non planifiée se caractérise par une augmentation des responsabilités du service informatique et par l'absence de moyens nécessaires pour établir des priorités ou encore pour cristalliser les projets en voie de développement. Lors de la phase de contrôle, la direction conclut souvent que la seule façon d'assurer un meilleur contrôle de ses ressources est de prendre des mesures draconiennes. C'est souvent à ce moment que sont instaurés des mécanismes, tels que la facturation des services informatiques, l'imposition de normes et de méthodes pour supporter le développement d'applications informatiques ainsi que la mise en place de standards sur le plan de la programmation, de la documentation et des opérations. Finalement, la phase d'intégration est caractérisée par un raffinement des mesures de contrôle permettant de mieux exploiter la technologie disponible sans affecter les coûts. C'est lors de cette phase que l'on assiste à une rationalisation des opérations, à la mise en place d'analyses économiques permettant d'établir les priorités de développement ainsi qu'à une décentralisation des informaticiens dans les différents départements utilisateurs.

Examiné sous cet angle, le modèle de Nolan constitua un apport important en ce qui concerne l'évolution des SIO, en intégrant non seulement les aspects liés à l'utilisation des SIO (types de technologies utilisées, composition du portefeuille d'applications), mais également ceux liés à leur gestion (planification, organisation et contrôle). Il constitua du même coup un apport considérable sur le plan de la caractérisation des SIO avec l'introduction d'un concept relativement nouveau et important dans ce domaine, soit celui de la " maturité » des organisations en regard de leurs systèmes d'information. Le modèle étapiste de Nolan, a en réalité introduit la notion de maturité informatique du fait qu'il existe un lien étroit entre cette notion et celle associée à l'évolution des SIO. Nolan (1979) perçoit la maturité comme étant le stade ultime de la croissance informatique des organisations; stade caractérisé par le plein développement des ressources informatiques de l'organisation.

À la suite de la présentation de la dernière version du modèle de Nolan, soit celle de 1979, un certain nombre de chercheurs commencèrent à s'intéresser à la complexité des SIO et plus particulièrement aux différents critères de " maturité » ou de " sophistication » des SIO (Benbasat et al., 1980; Cheney et Dickson, 1982 ; Ein-Dor et Segev, 1982 ; Lehman, 1985 ; Raymond, 1988). Malgré le fait que les concepts de "maturité » et de "sophistication » aient une même approche de caractérisation de la TI, dans le cadre de la présente recherche, nous avons préféré le terme "sophistication " à celui de " maturité ", à cause de l'association trop étroite de ce dernier terme à l'un des stades de la croissance informatique des organisations. 
Précisons que les études récentes ont élargi le concept de SIO utilisé à l'époque par Nolan et constitué uniquement d'applications administratives. En effet, un nombre considérable d'études réalisées durant la dernière décennie (Huff et Munro, 1985 ; Crowston et Treacy, 1986 ; Shanks, 1988 ; Cragg, 1989 ; Attewell, 1989 ; Blili et Rivard, 1990) parle désormais de technologie de l'information (TI) plutôt que de SIO, cette TI intégrant les utilisations informatiques et apparues durant la dernière décennie, telles l'informatisation de la production et les applications spécialisées de bureautique et de télématique.

Sous l'influence du modèle de Nolan (1979) ainsi que d'autres travaux de recherche ayant traité des concepts de "maturité » et de "sophistication » des SIO (Benbasat et al., 1980 ; Ein-Dor et Segev, 1982 ; Raymond, 1988), il paraît pertinent d'aborder la notion de sophistication de la TI à partir de deux perspectives distinctes. En effet, nous étudierons ce concept au regard de l'utilisation et de la gestion de la TI. La figure 1 présente le cadre général d'étude associé au concept de sophistication de la TI.

FIGURE 1

Dimensions de la sophistication de la TI

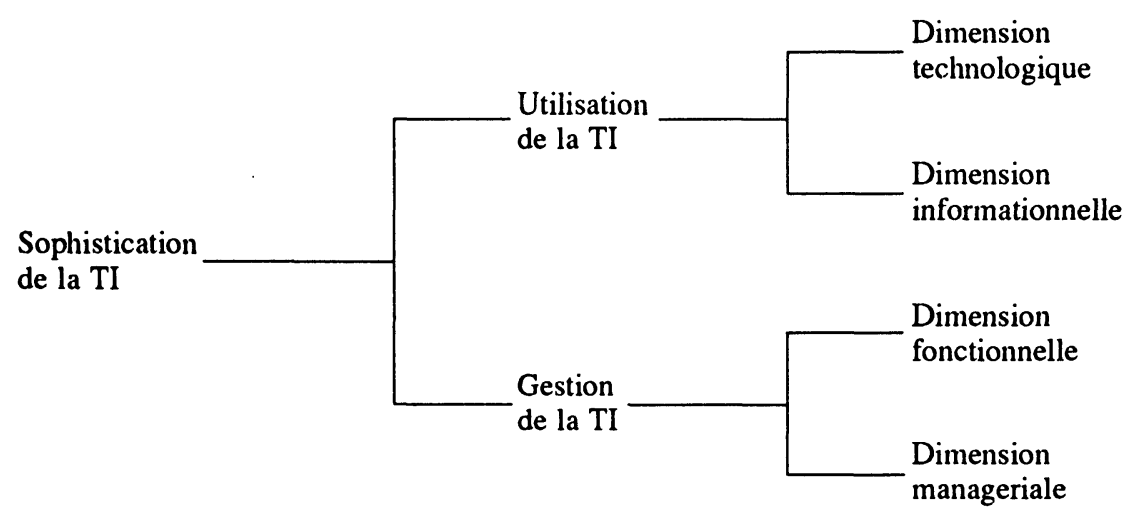

Cette caractérisation multidimensionnelle de la TI est importante, puisqu'elle nous permettra de déterminer plus facilement la présence de profils types de sophistication dans les organisations de petite et de moyenne dimension. Par ailleurs, la spécificité de la petite entreprise ainsi que celle des facteurs environnementaux, tels que la baisse des coûts du matériel, l'innovation technologique de la dernière décennie ainsi que la simplification dans l'utilisation des systèmes informatisés (Barcet et al., 1984), affecteront à des degrés divers chacune des quatre dimensions. Le tableau 1 présente les variables indicatrices de sophistication de la TI associées au contexte spécifique de la PME. Leur 
présence s'explique entre autres par leur caractère fondamental en tant que descripteur de la TI et par leur pertinence dans le contexte spécifique de la PME. En fait, ces variables recoupent l'ensemble des dimensions de la sophistication de la TI et elles ont préalablement fait l'objet d'études empiriques en contexte spécifique de PME.

\section{TABLEAU 1}

Variables indicatrices de sophistication de la TI en contexte PME

\begin{tabular}{|c|c|c|c|}
\hline Dimension & Variables indicatrices & Échelles & Types d'échelle \\
\hline Technologique & $\begin{array}{l}\text { Types de TI utilisées } \\
\text { Caractéristiques du matériel } \\
\text { Outils de développement } \\
\text { Interface des applications } \\
\text { Capacité de traitement } \\
\text { Type d'exploitation }\end{array}$ & $\begin{array}{c}1 \\
2-3 \\
4 \\
5 \\
6 \\
7\end{array}$ & $\begin{array}{l}\text { à intervalles } \\
\text { à intervalles } \\
\text { à intervalles } \\
\text { à intervalles } \\
\text { à intervalles } \\
\text { à intervalles }\end{array}$ \\
\hline Informationnelle & $\begin{array}{l}\text { Portefeuille d'applications } \\
\text { Intégration des SI }\end{array}$ & $\begin{array}{c}8 \\
9 \\
10\end{array}$ & $\begin{array}{l}\text { à intervalles } \\
\text { dichotomique } \\
\text { à intervalles }\end{array}$ \\
\hline Fonctionnelle & $\begin{array}{l}\text { Spécial. du personnel informatique } \\
\text { Rôle du service informatique } \\
\text { Envergure décisionnelle } \\
\text { Type de développement } \\
\text { Localisation du service informatique } \\
\text { Participation des utilisateurs }\end{array}$ & $\begin{array}{c}11 \\
12 \\
13 \\
14-16 \\
17 \\
18 \\
19-22\end{array}$ & $\begin{array}{l}\text { à intervalles } \\
\text { à intervalles } \\
\text { à intervalles } \\
\text { à intervalles } \\
\text { ordinale } \\
\text { dichotomique } \\
\text { dichotomique }\end{array}$ \\
\hline Manageriale & $\begin{array}{l}\text { Objectifs d'informatisation } \\
\text { Engagement de la haute direction } \\
\text { Budget informatique } \\
\text { Démarche d'informatisation } \\
\text { Recours à des consultants } \\
\text { Planification des SI } \\
\text { Contrôle des SI } \\
\text { Evaluation des SI }\end{array}$ & $\begin{array}{l}23-25 \\
26 \\
27-28 \\
29 \\
30 \\
31 \\
32 \\
33-35 \\
36-37\end{array}$ & $\begin{array}{l}\text { à intervalles } \\
\text { à intervalles } \\
\text { ordinale } \\
\text { à intervalles } \\
\text { à intervalles } \\
\text { à intervalles } \\
\text { ordinale } \\
\text { ordinale } \\
\text { dichotomique }\end{array}$ \\
\hline
\end{tabular}

\section{$\square$ Sophistication technologique de la TI}

Cette dimension reflète principalement le type de TI utilisées dans les entreprises de petite et moyenne dimension. Cette typologie fait référence de façon plus précise aux domaines de l'informatique de gestion, de l'informatique de production, de la bureautique ainsi que de la télématique (Lehman, 1985 ; Turner, 
1981 ; Grisé, 1989). À chacune de ces TI est associé un certain nombre d'applications informatiques caractérisées par un degré de sophistication plus ou moins élevé selon le type d'application.

La dimension technologique s'intéresse également aux caractéristiques du matériel et des outils de développement utilisés en contexte PME. En ce qui a trait au matériel, on fait référence principalement à la typologie des différents équipements informatiques (micro-ordinateurs, mini-ordinateurs, ordinateurs centraux) en place (Ein-Dor et Segev, 1982 ; Lehman, 1985 ; Cragg, 1986) ainsi qu'au degré de décentralisation des postes de travail (terminaux, micro-ordinateurs) à travers l'organisation (Turner, 1981 ; Cheney et Dickson, 1982). Pour ce qui est des outils de développement utilisés, on s'intéresse à la présence ainsi qu'au degré de sophistication des langages de programmation évolués et très évolués ( $3^{\mathrm{e}}$ et $4^{\mathrm{e}}$ génération) ainsi que des logiciels commerciaux (ex.: graphisme) utilisés (Cheney et Dickson, 1982; Alloway et Quillard, 1983 ; Lehman, 1985 ; Cragg, 1986). En outre, la dimension technologique s'intéresse au degré de sophistication de l'interface humain-machine associée aux principales applications informatiques (Lehman, 1985). Finalement, on aborde la notion de sophistication technologique sous l'angle de la capacité de traitement (en lots/en dialugué) des systèmes informatisés ainsi que sous celui du type d'exploitation (interne/externe) privilégié par les petites entreprises (Turner, 1981 ; Alloway et Quillard, 1983 ; Lehman, 1985 ; Cragg, 1986 ; Raymond, 1988).

\section{Sophistication informationnelle de la TI}

L'environnement informationnel d'une petite organisation au regard de sa TI est caractérisé principalement par la composition de son portefeuille d'applications qui peut être constitué d'applications administratives (ex. : paie, comptesclients, facturation, traitement de texte) et manageriales (ex. : gestion des stocks, échéancier de production, prix de revient, budgétisation) (Alloway et Quillard, 1983 ; Cragg, 1989). Toutefois, la documentation spécialisée nous incite à intégrer un élément additionnel à la dimension informationnelle : le degré d'intégration des applications informatiques (Ein-Dor et Segev, 1982). En contexte PME, cet élément est caractérisé habituellement par la présence d'une base de données centrale (données partagées par plusieurs applications) ainsi que par la mise en place d'un réseau de communication local.

\section{Sophistication fonctionnelle de la TI}

La dimension fonctionnelle s'intéresse à la fois aux aspects structurels du service informatique ou de la fonction SI des PME ainsi qu'aux aspects liés au développement et à l'implantation des applications informatiques. Ainsi, cette 
dimension s'intéresse dans un premier temps à la localisation du service informatique au sein de l'entreprise (Ein-Dor et Segev, 1982 ; Raymond, 1988). Par localisation, il faut entendre le niveau hiérarchique et le poste occupé dans l'organisation par le responsable de l'informatique ainsi que par son supérieur immédiat. Le degré de spécialisation du personnel informatique en place (EinDor et Segev, 1982 ; Cheney et Dickson, 1982 ; McFarlan et al., 1983 ; Lehman, 1985), à savoir le nombre de programmeurs, de programmeurs/analystes et d'analystes travaillant au sein de l'organisation constitue également une variable indicatrice de sophistication fonctionnelle. Par ailleurs, le degré de sophistication associé à cette dimension sera affecté par la diversité des rôles associés au service informatique (Benbasat et al., 1980 ; Cragg, 1986).

En rapport avec la hiérarchie des activités de management, on s'intéresse à l'envergure décisionnelle des applications informatiques en identifiant le pourcentage d'entre elles visant les opérations, le contrôle de gestion et la planification stratégique (Turner, 1981 ; Raymond, 1988). Finalement, au regard du développement et de l'implantation des applications informatiques, cette dimension s'intéresse au degré de formalisme associé à l'application d'une démarche systématique de développement, à l'importance du développement d'applications effeciué à l'interne, à l'importance des applications développées sur mesure ainsi qu'au degré d'engagement des utilisateurs lors du développement et de l'implantation des SIO (Benbasat et al., 1980).

\section{$\square$ Sophistication managériale de la TI}

La dimension manageriale s'intéresse principalement aux mécanismes de planification, de contrôle et d'évaluation des applications informatiques actuelles et futures (Benbasat et al., 1980 ; Cheney et Dickson, 1982).

Au regard des mécanismes de planification instaurés dans les PME, il s'avère pertinent, étant donné leur spécificité, de s'intéresser, dans une optique de sophistication, à des aspects particuliers tels le degré de formalisme, le degré de conformité aux objectifs organisationnels ainsi que l'identification de la ou des personnes responsables de la planification informatique au sein de l'organisation. La présence de documents, de normes, de procédés et de mesures sécuritaires relatifs au développement, à l'implantation et à l'utilisation des applications informatiques constitue également un indice d'une plus grande sophistication de la TI. La ventilation des dépenses informatiques engagées par la firme ainsi que la mise en place d'une évaluation post-implantation des SIO au sein de l'organisation constituent également des indications d'une plus grande sophistication sur le plan de la gestion de la TI en contexte de PME. 
Outre ces pratiques manageriales, ce type de sophistication est également influencé par les objectifs ainsi que par la démarche qui sous-tendent l'adoption de la TI dans l'entreprise, l'engagement des membres de la direction (Delone, 1988), le recours à des consultants externes (Miller, 1987 ; Montazemi, 1988), l'importance des sommes investies lors de l'informatisation initiale ainsi que les sommes consacrées annuellement au développement et à l'exploitation des applications informatiques (Montazemi, 1987).

\section{Méthodologie}

La population cible de l'étude regroupait l'ensemble des PME manufacturières de la province de Québec dont le nombre d'employés était compris entre 20 et 249. Les firmes artisanales furent exclues dans le but d'accroître la probabilité d'échantillonner des firmes informatisées, étant donné qu'on ne savait pas à l'avance si une firme était informatisée ou non. À l'aide d'un répertoire gouvernemental indiquant le nom et le nombre d'employés de toutes les entreprises manufacturières québécoises, un questionnaire fut envoyé à 1000 organisations.

Plusieurs études (Rumberger et Levin,1986; Julien et al., 1988) ayant confirmé le rôle dominant que jouent les propriétaires-dirigeants de PME sur le plan du processus d'informatisation de leur organisation, le questionnaire fut adressé au PDG de chacune des entreprises échantillonnées. On demandait à ce dernier de remplir la première partie du questionnaire portant notamment sur le processus d'informatisation de la firme. Une fois le questionnaire rempli, on demandait au président de le transmettre à la personne responsable de l'informatique au sein de l'entreprise. Nous avions précisé dans la lettre de présentation que cette personne devait occuper le poste hiérarchique le plus élevé en ce qui a trait à la fonction informatique, ou avait les plus grandes responsabilités en matière informatique au sein de l'entreprise. Il pouvait donc s'agir, selon l'entreprise, du PDG lui-même, du directeur du service informatique ou de la fonction SI, du chef-comptable, du contrôleur ou encore d'une secrétaire. Cette seconde partie du questionnaire comportait des questions ayant trait aux quatre dimensions du concept de sophistication de la TI. Advenant le cas où une entreprise ne disposait d'aucun apport informatique, le PDG devait alors cocher la case appropriée sur la page frontispice du questionnaire et nous retourner celuici non rempli dans l'enveloppe-réponse déjà affranchie.

À la suite de cet envoi postal, 173 questionnaires nous furent retournés, dont 122 provenaient de firmes informatisées et 51 , de firmes non informatisées. Les résultats de la présente étude seront toutefois basés sur les réponses de 101 firmes informatisées puisque parmi les 122 firmes de ce groupe, 21 d'entre 
elles ne satisfaisaient plus au critère typologique de taille. Ces firmes ont signifié avoir un nombre d'employés supérieur à 249 ; il s'agissait pour la plupart de filiales de grandes entreprises.

\section{Résultats}

À partir du cadre conceptuel présenté au tableau 1, 37 échelles de sophistication de la TI en contexte PME ont été retenues. La description associée à chacune de ces échelles de sophistication est présentée au tableau 2.

TABLEAU 2

\section{Échelles de sophistication de la TI en contexte PME}

\begin{aligned} \hline Échelle & \multicolumn{1}{c}{ Description } \\ \hline 1 & Nombre de technologies de l'information utilisées \\ 2 & Nombre de postes de travail utilisés (terminaux, micro-ordinateurs) \\ 3 & Degré de centralisation des équipements informatiques \\ 4 & Nombre de langages de programmation et de logiciels commerciaux utilisés \\ 5 & Qualité de l'interface des principales applications informatiques \\ 6 & Pourcentage des applications en mode dialogué \\ 7 & Pourcentage des applications exploitées à l'interne \\ 8 & Nombre d'applications de type administratif \\ 9 & Présence ou non d'un réseau de communication local \\ 10 & Pourcentage des applications dont les données sont intégrées à une banque de \\ & données centrale \\ 11 & Nombre de spécialistes informatiques \\ 12 & Nombre de responsabilités devant être assumées par la fonction SI \\ 13 & Pourcentage des applications visant le contrôle de gestion et la planification \\ & stratégique \\ 14 & Degré de formalisme lié à l'application d'une démarche de développement \\ 15 & Pourcentage des applications dont le développement a été effectué à l'interne \\ 16 & Pourcentage des applications dont le développement a été effectué sur mesure \\ 17 & Niveau hiérarchique du responsable informatique \\ 18 & Degré d'autonomie de la fonction SI \\ 19 & Participation ou non des utilisateurs à la phase d'analyse \\ 20 & Participation ou non des utilisateurs à l'élaboration d'un échéancier \\ 21 & Participation ou non des utilisateurs à l'élaboration d'un budget \\ 22 & Les utilisateurs sont ou ne sont pas des membres actifs de l'équipe de \\ 23 & développement \\ 24 & Importance des objectifs d'informatisation de niveau opérationnel \\ 25 & Importance des objectifs d'informatisation de niveau managerial \\ 26 & Attitude et engagement de la haute direction face à l'informatisation de la \\ 27 & firme \\ & Montant des investissements initiaux \end{aligned}


TABleaU 2 (suite)

Échelles de sophistication de la TI en contexte PME

\begin{tabular}{cl}
\hline Échelle & \multicolumn{1}{c}{ Description } \\
\hline 28 & Montant des dépenses annuelles \\
29 & Degré de formalisme associé à la démarche d'informatisation initiale \\
30 & Degré d'autonomie face à son environnement (recours à des consultants) \\
31 & Degré de formalisme du processus de planification informatique \\
32 & Nature du comité responsable de la planification informatique \\
33 & Nombre de documents supportant l'implantation et l'utilisation des applications \\
34 & Nombre de normes, procédés et standards implantés \\
35 & Nombre de mesures sécuritaires en place \\
36 & Ventilation ou non des dépenses informatiques \\
37 & Application ou non d'une évaluation postimplantation des applications \\
& informatiques \\
\hline
\end{tabular}

La fidélité de l'instrument fut testée à l'aide du coefficient alpha de Cronbach calculé pour les dimensions technologique (7 échelles), informationnelle (3 échelles), fonctionnelle (12 échelles), manageriale (15 échelles), ainsi que pour la mesure globale de sophistication (37 échelles). Ce coefficient a été jugé acceptable à partir de 0,65 et satisfaisant à partir de 0,80 (Nunally, 1978). La validité de contenu fut mesurée à partir des coefficients de corrélation $r$ de Pearson. Mentionnons que des coefficients positifs et significatifs à 0,05 ont été jugés comme indicateurs de la présence d'une validité de contenu (Straub, 1989). Les tableaux 3 à 6 présentent les résultats relatifs à la fidélité et à la validité de chacune des quatre dimensions.

TABLEAU 3

Fidélité et validité de la dimension technologique

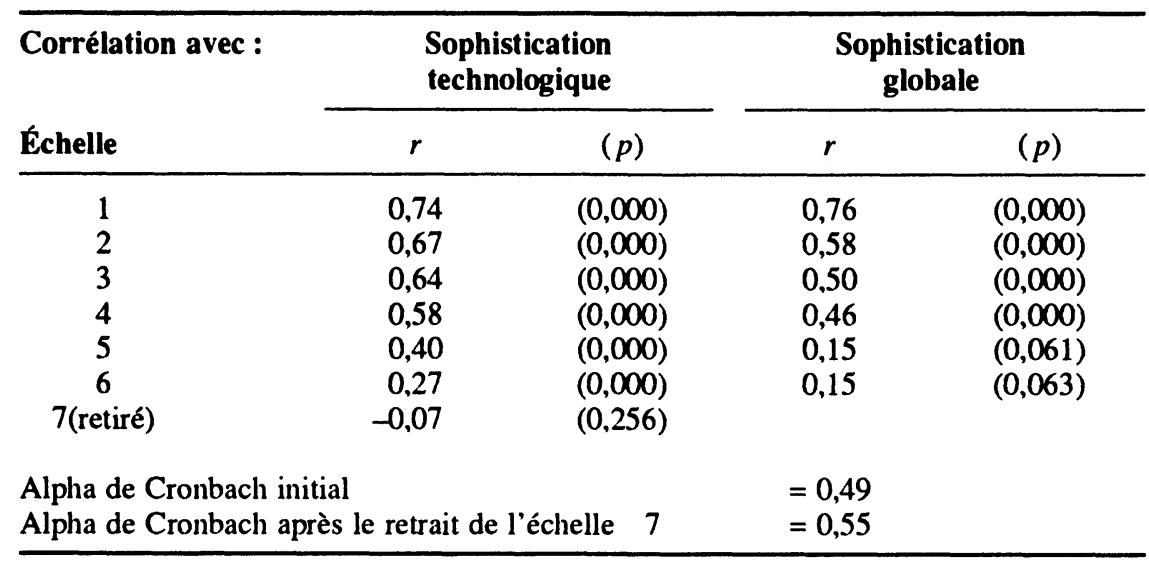


Tablead 4

Fidélité et validité de la dimension informationnelle

\begin{tabular}{ccccccc}
\hline Corrélation avec : & \multicolumn{2}{c}{$\begin{array}{c}\text { Sophistication } \\
\text { informationnelle }\end{array}$} & & \multicolumn{2}{c}{$\begin{array}{c}\text { Sophistication } \\
\text { globale }\end{array}$} \\
\cline { 2 - 3 } \cline { 5 - 6 } Échelle & $r$ & $(p)$ & & $r$ & $(p)$ \\
\hline 9 & 0,76 & $(0,000)$ & & 0,51 & $(0,000)$ \\
8 & 0,69 & $(0,000)$ & & 0,70 & $(0,000)$ \\
10 & 0,66 & $(0,000)$ & & 0,42 & $(0,000)$
\end{tabular}

Alpha de Cronbach $=0,50$

TABLEAU 5

Fidélité et validité de la dimension fonctionnelle

\begin{tabular}{cccccc}
\hline Corrélation avec : & \multicolumn{2}{c}{$\begin{array}{c}\text { Sophistication } \\
\text { fonctionnelle }\end{array}$} & & \multicolumn{2}{c}{$\begin{array}{c}\text { Sophistication } \\
\text { globale }\end{array}$} \\
\cline { 2 - 3 } \cline { 5 - 6 } Echelle & $r$ & $(p)$ & & $r$ & $(p)$ \\
\hline 22 & 0,61 & $(0,000)$ & & 0,35 & $(0,000)$ \\
12 & 0,60 & $(0,000)$ & & 0,55 & $(0,000)$ \\
15 & 0,56 & $(0,000)$ & & 0,38 & $(0,000)$ \\
11 & 0,54 & $(0,000)$ & & 0,29 & $(0,002)$ \\
20 & 0,53 & $(0,000)$ & & 0,39 & $(0,000)$ \\
14 & 0,52 & $(0,000)$ & & 0,31 & $(0,001)$ \\
21 & 0,47 & $(0,000)$ & & 0,34 & $(0,000)$ \\
19 & 0,39 & $(0,000)$ & & 0,33 & $(0,000)$ \\
17 & 0,38 & $(0,000)$ & & 0,23 & $(0,011)$ \\
13 & 0,32 & $(0,001)$ & & 0,14 & $(0,079)$ \\
18 & 0,32 & $(0,001)$ & & 0,17 & $(0,043)$ \\
16 (retiré) & 0,09 & $(0,186)$ & & & \\
& & & & & \\
Alpha de Cronbach initial & & & & \\
Alpha de Cronbach après le retrait de l'échelle 16 & $=0,65$ & \\
\hline
\end{tabular}


TABLEAU 6

Fidélité et validité de la dimension managériale

\begin{tabular}{cccccc}
\hline Corrélation avec : & \multicolumn{2}{c}{$\begin{array}{c}\text { Sophistication } \\
\text { manageriale }\end{array}$} & & \multicolumn{2}{c}{$\begin{array}{c}\text { Sophistication } \\
\text { globale }\end{array}$} \\
\cline { 2 - 3 } \cline { 5 - 6 } Échelle & $r$ & $(p)$ & & $r$ & $(p)$ \\
\hline 27 & 0,68 & $(0,000)$ & & 0,67 & $(0,000)$ \\
34 & 0,66 & $(0,000)$ & & 0,58 & $(0,000)$ \\
33 & 0,64 & $(0,000)$ & & 0,55 & $(0,000)$ \\
35 & 0,60 & $(0,000)$ & & 0,48 & $(0,000)$ \\
29 & 0,58 & $(0,000)$ & & 0,48 & $(0,000)$ \\
31 & 0,54 & $(0,000)$ & & 0,39 & $(0,000)$ \\
36 & 0,50 & $(0,000)$ & & 0,41 & $(0,000)$ \\
23 & 0,48 & $(0,000)$ & & 0,27 & $(0,003)$ \\
37 & 0,46 & $(0,000)$ & & 0,34 & $(0,000)$ \\
25 & 0,42 & $(0,000)$ & & 0,29 & $(0,002)$ \\
30 & 0,40 & $(0,000)$ & & 0,18 & $(0,035)$ \\
24 & 0,40 & $(0,000)$ & & 0,29 & $(0,002)$ \\
28 & 0,34 & $(0,000)$ & & 0,53 & $(0,000)$ \\
32 & 0,27 & $(0,003)$ & & 0,20 & $(0,021)$ \\
26 & 0,23 & $(0,011)$ & & 0,07 & $(0,249)$ \\
& & & & \\
Alpha de Cronbach & $=0,77$ & & &
\end{tabular}

On constate que la structure proposée en regard de chacune des dimensions est essentiellement correcte, étant donné la présence de relations positives et significatives entre le score global associé à chaque dimension (moyenne des échelles de la dimension) et chacune des échelles qui compose cette même dimension. En fait, seules les échelles 7 (\% des applications exploitées à l'interne) et 16 (\% des applications dont le développement a été effectué sur mesure) n'ont pas présenté des résultats significatifs.

En ce qui a trait à l'échelle 7, les résultats confirment ceux de Raymond (1988) selon lesquels le type d'exploitation ne semble pas constituer une variable indicatrice de sophistication. En fait, les progrès technologiques de la dernière décennie associés à l'avènement de la micro-informatique, conjugués à des baisses spectaculaires dans le coût du matériel ainsi qu'à la disponibilité sur le marché de logiciels d'application de plus en plus flexibles et conviviaux, permettent à la petite et moyenne entreprise d'être exploitée à l'aide du personnel en place (non spécialisé). Ce constat explique, du moins en partie, la tendance marquée des petites firmes à opter initialement pour le traitement à l'interne. 
Pour sa part, la non-pertinence de l'échelle 16 peut être expliquée, du moins partiellement, par l'avènement de la micro-informatique conjugué à des progrès technologiques considérables en ce qui concerne les logiciels d'application en vente sur le marché. En effet, de nombreuses études empiriques ont montré que les petites firmes manquent souvent de ressources financières et humaines nécessaires au développement d'applications sur mesure. Ces firmes doivent donc acquérir des progiciels standard ou modifiés provenant de fournisseurs ou de centres de traitement à façon (Montazemi, 1987). Par ailleurs, les progrès technologiques récents dans le domaine de la micro-informatique couplés à du logiciel d'exploitation et d'application plus flexible et sophistiqué permettent désormais de développer, outre les applications généralisables à plusieurs organisations, des applications de type managerial où les besoins en information deviennent plus spécifiques à chaque firme (ex.: prix de revient, gestion des commandes, analyses financières, gestion de projets). Ainsi, le recours à des spécialistes externes ou à du personnel informatique interne afin de concevoir des applications sur mesure devient moins indispensable même pour la petite entreprise.

À la lumière des résultats présentés aux tableaux 3 à 6 , nous avons retranché, dvant de poursuivre notre analyse de fidélité et de validité, les deux échelles (7 et 16) ayant présenté des résultats peu concluants du point de vue de la validité de leur contenu. Nous obtenons ainsi un instrument comportant 35 échelles de sophistication. Les analyses qui suivent feront référence à l'instrument amélioré.

La validité de construit de l'instrument fut testée, dans un premier temps, en présentant les coefficients de corrélation existant entre chacune des échelles et la mesure globale de sophistication (réf. tableaux 3 à 6). Parmi les 35 échelles de sophistication, seule l'échelle 26 (engagement de la haute direction) n'a pas présenté des résultats significatifs $(r=0,07$ et $p=0,25)$. Toutefois, considérant les résultats satisfaisants obtenus sur le plan de la validité de contenu pour la dimension manageriale $(r=0,23$ et $p=0,01)$, nous avons décidé de conserver cette échelle dans l'instrument de mesure. La validité de construit fut également testée en corrélant chaque dimension (moyenne des échelles) avec la mesure globale de sophistication (moyenne des 37 échelles) ainsi qu'avec la mesure globale ajustée (moyenne des échelles excluant celles associées à la dimension corrélée). Le tableau 7 présente ces résultats ainsi que les coefficients de corrélation interdimensions qui supportent également la validité de construit de l'instrument. Il est important de souligner qu'une analyse factorielle n'a pu être effectuée afin de supporter la validité de construit de l'instrument de mesure, le ratio taille de l'échantillon/nombre d'échelles étant inférieur à 3/1. En ce qui a trait à la fidélité de l'instrument de mesure amélioré, mentionnons que le coefficient alpha de Cronbach fut jugé satisfaisant, ce dernier ayant présenté un résultat de 0,84 . 
TABLEAU 7

Validité de construit de la sophistication de la TI

\begin{tabular}{|c|c|c|c|c|}
\hline Corrélation avec: & $\begin{array}{l}\text { Sophistication } \\
\text { technologique }\end{array}$ & $\begin{array}{l}\text { Sophistication } \\
\text { informationnelle }\end{array}$ & $\begin{array}{l}\text { Sophistication } \\
\text { e fonctionnelle }\end{array}$ & $\begin{array}{c}\text { Sophistication } \\
\text { managériale }\end{array}$ \\
\hline Mesure globale & $0,80^{* * *}$ & $0,77^{* * *}$ & $0,70^{* * *}$ & $0,74^{* * *}$ \\
\hline $\begin{array}{l}\text { Mesure globale } \\
\text { ajustée }\end{array}$ & $0,61 * * *$ & $0,49 * * *$ & $0,51^{* * *}$ & $0,57 * * *$ \\
\hline $\begin{array}{l}\text { Sophistication } \\
\text { technologique }\end{array}$ & - & & & \\
\hline $\begin{array}{l}\text { Sophistication } \\
\text { informationnelle }\end{array}$ & $0,45^{* * *}$ & - & & \\
\hline $\begin{array}{l}\text { Sophistication } \\
\text { fonctionnelle }\end{array}$ & $0,50^{* * *}$ & $0,26 * *$ & - & \\
\hline $\begin{array}{l}\text { Sophistication } \\
\text { manageriale }\end{array}$ & $0,45^{* *}$ & $0,45^{* *}$ & $0.49 * * *$ & - \\
\hline \multicolumn{5}{|c|}{$* * *=p<0,001$} \\
\hline
\end{tabular}

La validité prédictive d'un instrument pouvant être démontrée par la corrélation de cet instrument avec une mesure alternative du même construit, nous avons alors inclus dans l'instrument une mesure du construit inspirée des travaux de Nolan (1979). Cette mesure présente une brève description de chacun des stades d'évolution de l'informatique dans les organisations. Par ailleurs, sur la base des résultats d'études empiriques antérieures, on s'attend à ce que la taille d'une entreprise soit associée au degré de sophistication de sa TI (Rumberger et Levin, 1986 ; Delone, 1988). On s'attend aussi à ce que l'expérience informatique d'une entreprise soit directement reliée au degré de sophistication associé à sa TI, soit la validité de l'instrument sur le plan temporel (Benbasat et al., 1980 ; Raymond, 1988), l'expérience informatique de la firme étant mesurée par le nombre d'années depuis l'informatisation initiale. Enfin, on s'attend à ce que le degré d'éducation du PDG influence positivement le degré de sophistication informatique de la firme (Raymond, 1982). Le tableau 8 présente les résultats relatifs à la validité prédictive de l'instrument de mesure élaboré dans le cadre de la présente étude. 
TABLEAU 8

Validité prédictive de la sophistication de la TI

\begin{tabular}{llllll}
\hline $\begin{array}{l}\text { Corrélation } \\
\text { avec : }\end{array}$ & $\begin{array}{c}\text { Sophist. } \\
\text { globale }\end{array}$ & $\begin{array}{c}\text { Sophist. } \\
\text { techno. }\end{array}$ & $\begin{array}{c}\text { Sophist. } \\
\text { inform. }\end{array}$ & $\begin{array}{c}\text { Sophist. } \\
\text { fonction. }\end{array}$ & $\begin{array}{c}\text { Sophist. } \\
\text { manager. }\end{array}$ \\
\hline $\begin{array}{l}\text { Maturité } \\
\text { informatique }\end{array}$ & $0,35^{* * *}$ & $0,22^{* * *}$ & $0,27 * *$ & 0,15 & $0,41^{* * *}$ \\
$\begin{array}{l}\text { Expérience } \\
\text { informatique }\end{array}$ & $0,37^{* * *}$ & $0,44^{* * *}$ & 0,15 & $0,36^{* * *}$ & $0,19 *$ \\
$\begin{array}{l}\text { Taille de la } \\
\text { firme }\end{array}$ & $0,39 * * *$ & $0,27^{* *}$ & $0,34^{* * *}$ & $0,31^{* * *}$ & $0,24^{* *}$ \\
$\begin{array}{l}\text { Niveau } \\
\text { d'éducation } \\
\text { du PDG }\end{array}$ & $0,23^{*}$ & $0,26^{* *}$ & 0,02 & $0,26^{* *}$ & $0,21^{*}$ \\
& & & & &
\end{tabular}

$*=p<0,05 \quad * *=p<0,01 \quad * * *=p<0,001$

Sur la base des coefficients $r$ de Pearson, on peut confirmer la validité prédictive de l'instrument. En fait, ce tableau indique clairement que l'expérience informatique des firmes, la taille des entreprises ainsi que le niveau d'éducation des PDG sont des variables fortement corrélées avec le construit de sophistication de la TI, tel que défini dans le cadre de la présente étude. Par ailleurs, la mesure alternative du construit, soit celle associée à la maturité informatique des firmes (Nolan, 1979), a présenté des résultats tout aussi concluants, démontrant ainsi la validité prédictive de l'instrument de mesure.

\section{4. Élaboration de profils-types de sophistication de la TI en contexte PME}

L'un des objectifs sous-jacents à notre projet de recherche étant de caractériser la nature de la technologie de l'information dans une organisation de petite ou moyenne dimension, nous avons établi, à partir du cadre conceptuel élaboré dans cette étude et des résultats présentés jusqu'à maintenant, l'existence de profils-types de sophistication de la TI. Le tableau 9 présente, dans un premier temps, la caractérisation bipolaire associée à la sophistication technologique de la TI dans les PME. 
TABleau 9

Profil type de sophistication technologique en contexte PME

\begin{tabular}{|c|c|c|}
\hline Variables indicatrices & Profil simple & Profil complexe \\
\hline $\begin{array}{l}\text { Technologies de } \\
\text { l'information }\end{array}$ & $\begin{array}{l}\text { Limitées } \\
\text { (applications } \\
\text { traditionnelles) }\end{array}$ & $\begin{array}{c}\text { Diversifiées } \\
\text { (télécopie, SIAD, CAO } \\
\text { GPAO, réseau local) }\end{array}$ \\
\hline $\begin{array}{l}\text { Centralisation des } \\
\text { équipements }\end{array}$ & $\begin{array}{c}\text { Centralisés } \\
\text { (1 seul département) }\end{array}$ & $\begin{array}{c}\text { Décentralisés } \\
\text { (2 ou } 3 \text { départements) }\end{array}$ \\
\hline Matériel informatique & $\begin{array}{l}\text { Limité } \\
\text { (1 ou } 2 \text { micros }\end{array}$ & $\begin{array}{l}\text { Étendu } \\
\text { (micros et minis) }\end{array}$ \\
\hline Outils de développement & $\begin{array}{l}\text { Élémentaires } \\
\text { (tableur, progiciel } \\
\text { comptable, Basic) }\end{array}$ & $\begin{array}{c}\text { Sophistiqués } \\
\text { (SGBD, graphisme, } \\
\text { AUTOCAD) }\end{array}$ \\
\hline Capacité de traitement & $\begin{array}{c}\text { En lots } \\
\text { (rapports imprimés) }\end{array}$ & $\begin{array}{c}\text { En dialogué } \\
\text { (interrogations, analyses) }\end{array}$ \\
\hline Qualité de l'interface & $\begin{array}{c}\text { Faible } \\
\text { (écrans compliqués, verbeux, } \\
\text { pas de fonction d'aide, } \\
\text { langage de commandes) }\end{array}$ & $\begin{array}{c}\text { Haute } \\
\text { (écrans clair, concis, } \\
\text { fonction d'aide, } \\
\text { menus interactifs) }\end{array}$ \\
\hline
\end{tabular}

Un profil technologique « complexe " par opposition à un profil technologique « simple " en contexte spécifique de PME est associé à :

- l'utilisation de TI liées à la fois aux domaines de la productique, de la bureautique et de la télématique. De façon plus précise, un degré de sophistication technologique complexe en PME fait référence à l'utilisation de technologies telles que la télécopie, les systèmes interactifs d'aide à la décision (SIAD), les applications qui sous-tendent le processus de production telles que la gestion des achats, des stocks et des commandes, les applications de conception et de fabrication assistées par ordinateur, ainsi que l'échange électronique des données avec l'environnement de l'organisation (ex. : clients, fournisseurs) ;

- une décentralisation des équipements informatiques. En fait, un degré de sophistication technologique complexe en PME indique la présence d'équipements au sein de deux ou plusieurs départements dont ceux du marketing et de la production qui constituent le véritable « cœur » de l'entreprise, particulièrement dans le secteur manufacturier (Barcet et al., 1984) ; 
- la présence des ressources matérielles qui facilitent l'implantation d'applications nécessitant un degré de complexité technologique supérieur. Par exemple, la présence d'un mini-ordinateur favorise le développement d'applications relativement complexes qui sous-tendent les processus de production, de gestion des commandes et de facturation de l'entreprise manufacturière. Par ailleurs, la présence de quelques postes de travail autonomes (micro-ordinateurs) favorise l'utilisation, par les gestionnaires, de SIO plus évolués tels que les SIAD qui répondent aux besoins informationnels particuliers de ces derniers, tandis que la présence de micro-ordinateurs puissants et de logiciels commerciaux spécialisés favorise l'implantation d'applications liées aux domaines de la bureautique (informatique graphique, messagerie électronique) et de la télématique (réseau de communication local) ;

- l'utilisation d'outils de développement puissants et perfectionnés, lesquels facilitent l'intégration d'un plus grand nombre d'applications de type managerial. Par exemple, l'utilisation d'un système de gestion de bases de données permet une meilleure intégration d'applications (achats, commandes, stocks, analyses et prévisions financières, prix de revient). L'utilisation de logiciels spécialisés de CAO/FAO permet pour sa part une intégration complète du processus de production. Finalement, l'utilisation de logiciels de graphisme encourage à son tour le développement d'applications de support décisionnel par les gestionnaires ;

- la présence de capacités de traitement en mode dialogué. Cela peut s'expliquer par le fait que les applications supportant la prise de décision dans les domaines de la production et de la commercialisation requièrent des réponses plus rapides et une plus grande interaction humain-machine ;

- une qualité accrue de l'interface humain-machine. Les attitudes et les comportements des gestionnaires envers les applications manageriales sont en grande partie dictés par des facteurs tels que la convivialité et la flexibilité des applications, celles-ci devant s'adapter aux différents styles de recherche et de traitement de l'information des gestionnaires.

Le tableau 10 présente la caractérisation bipolaire relative à la sophistication informationnelle de la TI dans les PME. 
TABLEAU 10

Profil type de sophistication informationnelle en contexte PME

\begin{tabular}{|c|c|c|}
\hline Variables indicatrices & Profil administratif & Profil managerial \\
\hline Portefeuille d'applications & $\begin{array}{l}\text { Applications } \\
\text { administratives } \\
\text { (g/l, c/c, c/f, paie, } \\
\text { facturation) }\end{array}$ & $\begin{array}{c}\text { Applications } \\
\text { manageriales } \\
\text { (budgets, commandes, } \\
\text { prix de revient, finance, } \\
\text { personnel, achats, modélisation) }\end{array}$ \\
\hline $\begin{array}{l}\text { Intégration des applications } \\
\text { à une base de données } \\
\text { centrale }\end{array}$ & $\begin{array}{c}\text { Minorité } \\
\text { d'applications }\end{array}$ & $\begin{array}{c}\text { Majorité } \\
\text { d'applications }\end{array}$ \\
\hline $\begin{array}{l}\text { Présence d'un réseau de } \\
\text { communication local }\end{array}$ & Non & Oui \\
\hline
\end{tabular}

Un profil de sophistication informationnelle de type «managerial » par opposition à un profil de type « transactionnel » en contexte spécifique de PME est associé à :

- la présence d'applications dites manageriales orientées vers l'efficacité décisionnelle des gestionnaires plutôt que vers l'efficience opérationnelle. Des exemples de telles applications concernent entre autres les domaines de la finance (prévisions et analyses financières, budgétisation), de la production (planification et suivi de la production) et du marketing (prévisions des ventes);

- une plus grande intégration des applications informatiques en raison de la présence d'une base de données centrale et d'un réseau de communication local. D'une part, la présence d'une base de données centrale facilite l'intégration des applications administratives et manageriales du fait que logiquement les extrants des premières constituent les intrants des secondes; d'autre part, la présence d'un réseau de communication local encourage le partage de données entre les différentes fonctions et niveaux hiérarchiques de l'organisation.

Le tableau 11 présente la caractérisation bipolaire associée au profil de sophistication fonctionnelle de la TI en contexte de petite et moyenne entreprise. 
TABLEAU 11

Profil type de sophistication fonctionnelle en contexte PME

\begin{tabular}{|c|c|c|}
\hline Variables indicatrices & Profil dépendant & Profil autonome \\
\hline $\begin{array}{l}\text { Spécialisation du personnel } \\
\text { informatique }\end{array}$ & $\begin{array}{l}\text { Restreinte } \\
\text { (préposés à la saisie } \\
\text { opérateurs) }\end{array}$ & $\begin{array}{c}\text { Etendue } \\
\text { (programmeur } \\
\text { prog./analyste, analyste) }\end{array}$ \\
\hline $\begin{array}{l}\text { Rôles de la fonction } \\
\text { informatique (SI) }\end{array}$ & $\begin{array}{l}\text { Opérations } \\
\text { (exploitation des } \\
\text { applications) }\end{array}$ & $\begin{array}{c}\text { Gestion } \\
\text { (formation, planification, } \\
\text { développement, contrôle) }\end{array}$ \\
\hline $\begin{array}{l}\text { Application d'une démarche } \\
\text { systématique de développement }\end{array}$ & Rarement & Régulièrement \\
\hline Type de développement & Externe & Interne \\
\hline Envergure décisionnelle & Opérations & Contrôle de gestion \\
\hline $\begin{array}{l}\text { Localisation de la fonction } \\
\text { informatique (SI) }\end{array}$ & $\begin{array}{l}\text { Subordonnée à la } \\
\text { fonction comptable }\end{array}$ & Autonome \\
\hline $\begin{array}{l}\text { Participation des } \\
\text { utilisateurs }\end{array}$ & $\begin{array}{c}\text { Limitée } \\
\text { (analyse des besoins) }\end{array}$ & $\begin{array}{c}\text { Soutenue } \\
\text { (membres actifs de l'équipe } \\
\text { de développement) }\end{array}$ \\
\hline
\end{tabular}

Un profil fonctionnel « autonome », par opposition à un profil « dépendant » en contexte spécifique de PME, est associé à :

- la présence de personnel informatique spécialisé ayant les compétences suffisantes, compte tenu de l'environnement managerial et technologique plus complexe et changeant associé à l'implantation d'applications manageriales. Par personnel informatique spécialisé on entend, soit un programmeur, soit un programmeur-analyste ou un analyste ;

- un niveau décisionnel des applications informatiques orienté davantage vers le contrôle de gestion plutôt que vers l'efficience opérationnelle ;

- l'application soutenue d'une démarche systématique de développement des applications informatiques. L'application d'une méthode de développement permettra à l'entreprise d'obtenir un produit qui réponde aux besoins des utilisateurs, qui s'intègre bien aux activités de l'organisation et qui soit techniquement correct, tout en respectant les budgets et les échéances préalablement établis ;

- un niveau plus élevé de développement interne. Cela peut s'expliquer par le fait que l'information de support managerial ou décisionnel tend à être plus spécifique à chaque firme que le traitement transactionnel ; 
la nécessité de développer à l'interne se fait plus sentir à mesure que les requêtes pour des applications reliées à la production et au marketing augmentent en nombre ;

- la présence d'une fonction SI autonome. La nécessité de dissocier la gestion de la TI de la fonction comptable devient plus apparente au fur et à mesure que l'on intègre des applications propres aux domaines de la production et du marketing ;

- une participation soutenue des utilisateurs. Le développement d'applications visant le support décisionnel nécessite en fait un plus grand engagement des gestionnaires puisque ces applications supposent un plus grand changement dans la logique de gestion de l'organisation.

Finalement, le tableau 12 présente la caractérisation bipolaire associée au profil type de sophistication manageriale de la TI dans les PME.

\section{TABlEAU 12}

\section{Profil type de sophistication manageriale en contexte PME}

\begin{tabular}{|c|c|c|}
\hline Variables indicatrices & Profil réactif & Profil pro-actif \\
\hline Investissements en équipements & Minimes & Substantiels \\
\hline Dépenses annuelles & Minimes & Substantielles \\
\hline Démarche d'informatisation & Informelle & Formelle \\
\hline $\begin{array}{l}\text { Degré de formalisme du } \\
\text { processus de planification }\end{array}$ & Informel & Formel \\
\hline $\begin{array}{l}\text { Responsable de la } \\
\text { planification informatique }\end{array}$ & $\begin{array}{l}\text { Aucun responsable } \\
\text { formel }\end{array}$ & $\begin{array}{l}\text { Un membre de la } \\
\text { haute direction }\end{array}$ \\
\hline $\begin{array}{l}\text { Documentation des } \\
\text { applications informatiques }\end{array}$ & $\begin{array}{c}\text { Partielle } \\
\text { (provient de l'extérieur) }\end{array}$ & $\begin{array}{c}\text { Complète } \\
\text { (provient de l'intérieur) }\end{array}$ \\
\hline $\begin{array}{l}\text { Présence de normes, de } \\
\text { standards et de procédure }\end{array}$ & Informels & Formels \\
\hline $\begin{array}{l}\text { Présence de mesures } \\
\text { sécuritaires }\end{array}$ & $\begin{array}{l}\text { Limitées } \\
\text { (accès aux données, } \\
\text { copies de sécu/rité) }\end{array}$ & $\begin{array}{c}\text { Étendues } \\
\text { (validation à l'entrée, } \\
\text { accès aux équipements, } \\
\text { recouvrement fichiers) }\end{array}$ \\
\hline $\begin{array}{l}\text { Objectifs associés à } \\
\text { l'introduction de la TI }\end{array}$ & Informels & Formels \\
\hline Évaluation postimplantation & Non & Oui \\
\hline Ventilation des dépenses & Non & Oui \\
\hline Recours à des consultants & Dépendant & Autonome \\
\hline $\begin{array}{l}\text { Engagement et attitude de } \\
\text { la haute direction }\end{array}$ & Faible et indifférente & Forte et positive \\
\hline
\end{tabular}


Un profil managerial de type «pro-actif » par opposition à un profil de type "réactif » en contexte de PME est associé à :

- la présence de ressources financières appréciables allouées à la fonction informatique sans lesquelles l'entreprise ne pourra disposer des ressources humaines et matérielles lui permettant d'assurer une utilisation et une gestion adéquates de sa TI ;

- une démarche formelle lors de l'informatisation initiale. Cette démarche fait référence à une analyse des besoins informationnels, à la vérification auprès d'intervenants externes (concurrents, fournisseurs) ainsi qu'à une étude de faisabilité. Quelques-unes de ces entreprises iront jusqu'à l'élaboration d'un plan directeur :

- un processus de planification informatique formel et dont la responsabilité appartient à un ou à plusieurs membres de la direction. Une croissance ordonnée des systèmes d'information doit être planifiée et les ressources informationnelles allouées en conséquence. La complexité des SIO, l'interaction entre de nombreux éléments techniques et organisationnels, l'impact à long terme de tous les choix montrent l'intérêt d'une planification plus formelle ;

- un contrôle plus rigoureux des applications informatiques en place (documentation complète, normes, procédure et standards formels, mesures sécuritaires adéquates). En effet, la sophistication du portefeuille d'applications implique l'élaboration de pratiques et de techniques visant un contrôle adéquat pour la fonction SI ;

- la définition d'objectifs formels et dérivés des objectifs organisationnels. Les décisions prises à l'aide d'applications manageriales sont intimement reliées à la logique de gestion de l'organisation et, de ce fait, sont déterminantes à son succès ; l'utilisation de telles applications est donc potentiellement plus bénéfique, mais aussi plus risquée pour la PME. Il apparaît alors essentiel de gérer l'implantation graduelle du portefeuille d'applications en fonction des objectifs organisationnels ;

- la mise en application d'une évaluation postimplantation des applications informatiques. Il devient en fait plus important d'être en mesure d'évaluer l'efficacité des applications après implantation lorsque le portefeuille d'applications affecte les fonctions clés de l'organisation ;

- l'évaluation interne des services informatiques. La présence d'un tel système permet à l'entreprise de maîtriser ses coûts et de décentraliser les décisions d'emploi des ressources informatiques ; 
- une plus grande autonomie en ce qui a trait à l'utilisation et la gestion de ses applications informatiques minimisant ainsi le recours à des spécialistes externes ;

- une engagement actif et une attitude positive des dirigeants. Les applications manageriales devant s'adapter aux différents styles de recherche et de traitement de l'information des dirigeants, ces derniers se doivent de participer à part entière au processus de conception et d'implantation des applications et ils se doivent d'être sensibilisés au nouvel environnement technologique.

\section{Conclusion}

Les contributions de cette recherche sont de deux types : celles ayant trait à ses apports théoriques et conceptuels et celles qui concernent plus particulièrement son utilité pratique. Sur le plan conceptuel, cette étude constitue l'une des premières tentatives de caractérisation de la TI et permet d'élaborer une mesure fidèle et valide du construit de sophistication, axée à la fois sur l'utilisation et la gestion de la TI dans les PME. Or, les résultats de la présente étude constituent un premier pas vers une théorie descriptive de sophistication de la TI dans les PME. En fait, comme nous l'avons déjà mentionné, cette étude s'inscrit dans deux domaines de recherche particuliers soit les impacts organisationnels de l'informatisation et les méthodes appropriées de gestion de l'informatisation. L'utilisation de notre instrument de mesure par d'autres chercheurs permettra ainsi de démontrer l'impact de la technologie de l'information sur la structure, la stratégie et la performance des organisations de petite ou moyenne dimension.

Dans la pratique, l'instrument de mesure élaboré dans le cadre de cette étude peut être utilisé à diverses fins. Sur le plan pédagogique par exemple, cet instrument ainsi que le cadre théorique qui le sous-tend peuvent servir de base à l'élaboration de cours portant sur l'informatisation des PME. Sur le plan managerial, les contributions sont de deux types. En utilisant cet instrument pour mesurer l'impact de la TI sur les PME, la fidélité et la validité des résultats de recherches seront augmentées; de meilleures conclusions pourront être tirées. De plus, l'utilisation de cet instrument par des praticiens pour fins de diagnostic organisationnel permettra de mieux situer une entreprise par rapport aux autres sur le plan de l'utilisation et de la gestion de sa TI et ainsi de mieux définir les solutions proposées en fonction d'une meilleure connaissance de l'entreprise. 
La portée des résultats de cette étude est restreinte par un certain nombre de limites de nature méthodologique qu'il convient de considérer. La première limite associée à notre projet de recherche se situe sur le plan de la validité externe. En effet, l'échantillonnage fut réalisé dans un seul secteur d'activité, en l'occurrence le secteur manufacturier. En outre, le choix de la méthode de collecte des données, soit un questionnaire par envoi postal, implique possiblement un biais échantillonnal qui peut affecter la validité interne des résultats.

Dans le prolongement de cette étude, il serait pertinent de vérifier la présence de relations entre les profils types de sophistication de la TI, tels que définis dans le cadre de cette étude, et les pratiques actuelles d'utilisation et de gestion de la TI dans les PME. À la suite de l'étude de cette question, il sera alors possible de mieux évaluer le véritable impact de la sophistication croissante de la TI dans les PME, et ce, sur la structure, la stratégie et la performance de ces organisations.

\section{Bibliographie}

AllowaY, R.M. et J.A. QUillaRd (1983), «User managers' system needs », MIS Quarterly, vol. 7, n $^{\circ}$ 2, juin, p. 27-41.

Attewell, P., (1989), "Survey and other methodologies applied to information technology impact research », Workshop on Survey Research in MIS, University of California at Irvine, 10-11 février.

Barcet, A., J. Bonamy et A. Mayère (1984), «Informatique et PME : un dialogue en différé », Écononie et humanisme, 275, janvier-février, p. 44-56.

Benbasat, I., A.S. Dexter et R.W. Mantha (1980), «Impact of organizational maturity on information systems skill needs ", MIS Quarterly, vol. 4, $\mathrm{n}^{\circ} 1$, mars, p. 21-34.

Bengasat, I., Dexter, A.S., D.H. Drury et R.C. Goldsten,, (1984), " A critique of the stage hypothesis : theory and empirical evidence ", Communications of the $A C M$, vol. 27, $n^{\circ} 5$, mai, p. 476-485.

BERgERON, F. et C. ButEAU (1988), «Devancer la concurrence par les systèmes d'information ", Revue internationale PME, vol. 1, nos 3-4, p. 295-307.

Blidi, S. et S. Rivard (1990), « Technologies de l'information et stratégies d'entreprise : les prémisses d'une mutation », Teclinologies de l'information et société, vol. 2, $n^{\circ} 2$, p. $25-48$.

CHENEY, P.H. et G.W. Dickson (1982), «Organizational characteristics and information systems : an exploratory investigation », Academy of Management Journal, vol. 25, $\mathrm{n}^{\circ} 1$, mars, p. 170-184.

CRAGG, P. (1986), «A study of the impact of IT on the financial performance of small engineering firms ", Working Paper $n^{\circ} 132$, Loughborough University of Technology.

CRAGG, P. (1989), « Microcomputers and small firms in New Zealand », International Small Business Journal, vol. 5, $\mathrm{n}^{\circ} 1$, p. 41-45. 
Crowston, K. et M.E. Treacy (1986), « Assessing the impact of information technology on enterprise-level performance ", Proceedings of the $8^{\text {th }}$ International Conference on Information Systems, p. 299-310.

DELONE, W.H. (1988), « Determinants of success for computer usage in small business », MIS Quarterly, vol. 12, $\mathrm{n}^{\circ} 1$, mars, p. 51-62.

EIN-Dor, P. et E. SEgev (1982), «Organizational context and MIS structure: some empirical evidence ", MIS Quarterly, vol. 6, $\mathrm{n}^{\circ}$ 3, septembre, p. 55-68.

Grist, A., (1989), L'informatisation du Québec, profil de la demande, Les Publications du Québec, Québec.

HufF, S.L. et M.C. MunRo (1985), « Information technology assessment and adoption : a field study », MIS Quarterly, vol. 9, n 4, décembre, p. 327-340.

Julien, P.-A., J.-B. CARRIÉre et L. HÉBerT (1988), «Les facteurs de diffusion des nouvelles technologies dans les PME manufacturières », Revue internationale PME, vol. $1, \mathrm{n}^{\circ} 2$, p. 177-192.

Julien, P.-A. et M. Marchesnay (1988), La petite entreprise, Paris, Vuibert.

KING, J.L. et K.L. KRAEMER (1984), « Evolution and organizational information systems : an assessment of Nolan's stage model », Communications of the ACM, vol. 27, $\mathrm{n}^{\circ} 5$, p. $466-475$.

LehmaN, J.A. (1985), « Organizational size and information systems sophistication », Working Paper, $\mathrm{n}^{\circ} \mathrm{85}-18$, MIS Research Center, University of Minnesota.

McFarlan, F.W., J.L McKenney. et P. Pyburn (1983), "The information archipelago : plotting a course ", Harvard Business Review, vol. 61, $n^{\circ} 1$, janvier-février, p. 145-156.

MrLleR, D. (1987), «Strategy-making and structure : analysis and implications for performance ", Academy of Management Journal, vol. 30, $\mathrm{n}^{\circ} 1$, p. 7-32.

MintzBerg, H. (1979), The Structuring of Organizations, Englewood Cliffs, N.J., Prentice-Hall.

MonTAZEMI, A.R. (1987), " An analysis of IT assessment and adoption in small business environments ", INFOR, vol. 25, $\mathrm{n}^{\circ} 4$, p. 327-340.

MONTAZEMI, A.R., (1988), "Factors affecting information satisfaction in the context of the small business environment », MIS Quarterly, vol. 12, $n^{\circ} 2$, juin, p. 239-256.

Nolan, R.L., (1973), « Managing the computer resource : a stage hypothesis », Communications of the $A C M$, vol. $16, \mathrm{n}^{\circ} 7$, juillet, p. 399-405.

Nolan, R.L., (1979), "Managing the crises in data processing ", Harvard Business Review, vol. 57, $\mathrm{n}^{\circ}$ 2, mars-avril, p. 115-126.

Nunnaly, N. (1978), Psychometric Theory, New York, McGraw-Hill.

RAYMOND, L. (1982), " Problématique des systèmes d'information en contexte de PME », Congrès de l'ASAC, Ottawa, p. 51-61.

RAYMOND, L. (1988), « La sophistication des systèmes d'information en contexte PME : une approche par le portefeuille d'applications ", Canadian Journal of Administrative Sciences, vol.5, $\mathrm{n}^{\circ} 2$, p. 32-39.

RAYMOND, L. (1990), «Organizational context and information systems success: a contingency approach », Journal of MIS, vol. 6, $\mathrm{n}^{\circ} 4$, printemps, p. 5-20.

Rumberger, R.W. et H.M. Levin (1986), « Computers in small business », Institute for Enterprise Advancement, Washington, D.C. 
SHANKS, J.M. (1988), "Information technology and survey research : where do we go from here?», Computer-assisted Survey Methods Program, University of California, Berkeley, juin.

STRAUB, D.W. (1989), "Validating instruments in MIS research », MIS Quarterly, vol. 13, $\mathrm{n}^{\circ} 3$, juin, p. 13-18.

TURNER, J.A. (1981), "A method for measuring the properties of information systems ", Proceedings of the Third International Conference on Information Systems, p. 259-275. 